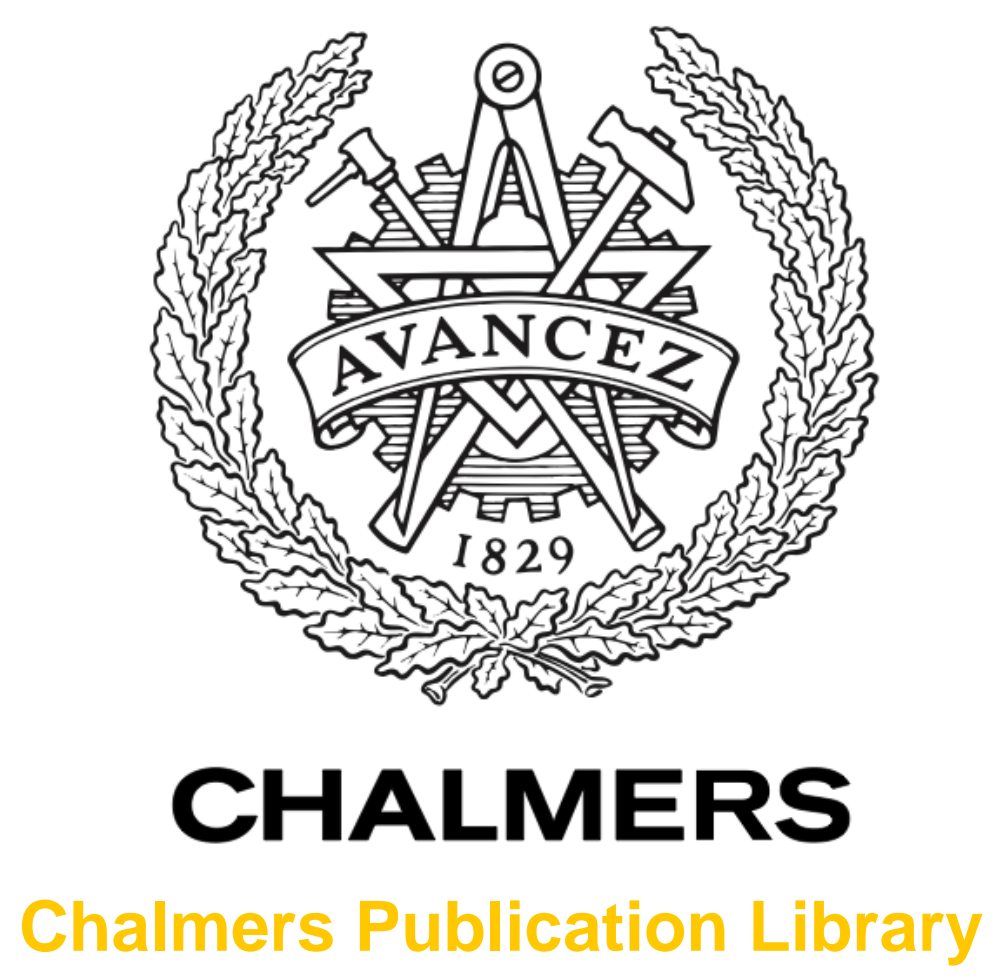

\title{
Experimental investigation of a 16-dimensional modulation format for long-haul multi-core fiber transmission
}

This document has been downloaded from Chalmers Publication Library (CPL). It is the author's version of a work that was accepted for publication in:

Proc. European Conference on Optical Communication (ECOC)

Citation for the published paper:

Rademacher, G. ; Puttnam, B. ; Luís, R. et al. (2015) "Experimental investigation of a 16dimensional modulation format for long-haul multi-core fiber transmission". Proc. European Conference on Optical Communication (ECOC)

Downloaded from: http://publications.lib.chalmers.se/publication/231108

Notice: Changes introduced as a result of publishing processes such as copy-editing and formatting may not be reflected in this document. For a definitive version of this work, please refer to the published source. Please note that access to the published version might require a subscription. 


\title{
Experimental Investigation of a 16-Dimensional Modulation Format for Long-Haul Multi-Core Fiber Transmission
}

\author{
G.Rademacher $^{(1,2)}$, B.J.Puttnam ${ }^{(1)}$, R.S.Luís ${ }^{(1)}$, Y.Awaji $^{(1)}$, N.Wada $^{(1)}$, E.Agrell $^{(3)}$, K.Petermann $^{(2)}$
}

(1) Photonic Network System Laboratory, National Institute of Information and Communications Technology (NICT), 4-2-1 Nukui-Kitamachi, Koganei, Tokyo 184-8759, Japan

(2) Technische Universität Berlin, Fachbereich Hochfrequenztechnik, Einsteinufer 25, 10587 Berlin, Germany Email: georg.rademacher@tu-berlin.de

(3) Dept. of Signals and Systems, Chalmers University of Technology, SE-412 96 Gothenburg, Sweden

Abstract We experimentally investigate a 16-dimensional modulation format applicable to multi-core fiber transmission, and demonstrate over $14,000 \mathrm{~km}$ transmission for a BER of $1 E-3$, a $55 \%$ improvement in reach compared to DP-BPSK for the same spectral efficiency.

\section{Introduction}

Space-division multiplexing (SDM) using multicore fibers (MCF) has recently been proposed as a technology to increase the per fiber capacity in future optical networks ${ }^{1}$. In addition to multiplying the transmission capacity, the relative uniformity of transmission characteristics of different cores in an $\mathrm{MCF}^{2}$ may also facilitate additional system benefits such as enabling transmission of multi-dimensional modulation formats ${ }^{3,4}$. Multidimensional formats can show improved noise and nonlinear performance compared to traditional four-dimensional (4-D) modulation formats such as dual-polarization quadrature phase shift keying (DP-QPSK) or binary phase shift keying (DP-BPSK) by considering optical modulation in multiple cores as being part of one multi-core symbol $^{5}$. The enhanced performance is provided by an increase of the minimum Euclidean distance $\left(d_{m i n}\right)$ between symbols. While optimizing of 4-D formats by considering $d_{\min }$ has been widely studied ${ }^{6}$, recent theoretical studies have shown that multi-dimensional modulation formats for up to 24 dimensions, based on higher-order lattices, block codes, or set-partitioning ${ }^{3-5}$ can have an even stronger sensitivity improvement without necessarily sacrificing spectral efficiency (SE). In single-core transmission, increased dimensionality for signal modulation has been investigated by joint modulation of timeslots ${ }^{4}$ and adjacent frequency channels ${ }^{7}$ but MCFs offer an opportunity to investigate such formats without sacrificing Baud rate or complicating receiver design. In this paper, we experimentally investigate a 16-dimensional modulation format optimized for transmission through four cores of a MCF. We demonstrate a $55 \%$ improvement in transmission distance at Bit Error Rate $(\mathrm{BER})=10^{-3}$ compared to DP-BPSK for the same SE and show that such formats may offer performance improvement in MCFs beyond linearly increasing capacity with the number of cores.

\section{Format Description and Implementation}

In this work, we have investigated a 16dimensional modulation format based on the extended Nordstrom-Robinson Code ${ }^{8,9}$, here referred to as $8 \mathrm{~B} 16 \mathrm{D}$. It has 256 symbols in 16 dimensions and can be used to transmit 8 bit per multi-core symbol through four cores, with equal spectral efficiency to DP-BPSK. As the Nordstrom-Robinson code is a nonlinear code, it cannot be obtained by multiplication of message words with a generator matrix. A simple construction method is given in ref. ${ }^{8}$ and the 256 codewords are listed in ref. ${ }^{9}$. The theoretical sensitivity at high optical signal to noise ratio (OSNR) for this format may be determined from the asymptotic power efficiency for the additive white Gaussian noise (AWGN) channel given by $\gamma=d_{\text {min }}^{2} \log _{2}(M) /\left(4 E_{S}\right)$, where $M$ is the number of constellation points and $E_{S}$ is the symbol energy. For a comparison applicable to MCF transmission, we define the SE normalized to each fiber core as $S E_{\text {core }}=\log _{2}(M) / 4 N$, where $N$ is the number of cores.

Tab. 1: Comparison of DP-BPSK / DP-QPSK and the investigated 16-dimensional modulation format.

\begin{tabular}{|l|c|c|c|c|} 
Name & $M$ & $d_{\min }^{2} / E_{s}$ & $\gamma(\mathrm{dB})$ & $S E_{\text {core }}$ \\
\hline DP-QPSK & 16 & 1 & 0 & 1 \\
DP-BPSK & 4 & 2 & 0 & 0.5 \\
8B16D & 256 & 1.5 & 4.77 & 0.5
\end{tabular}

As shown in Table 1, the 8B16D format has the same $S E_{\text {core }}$ as DP-BPSK but the larger $d_{\text {min }}$ provides a $4.77 d B$ increase in $\gamma$, indicat- 


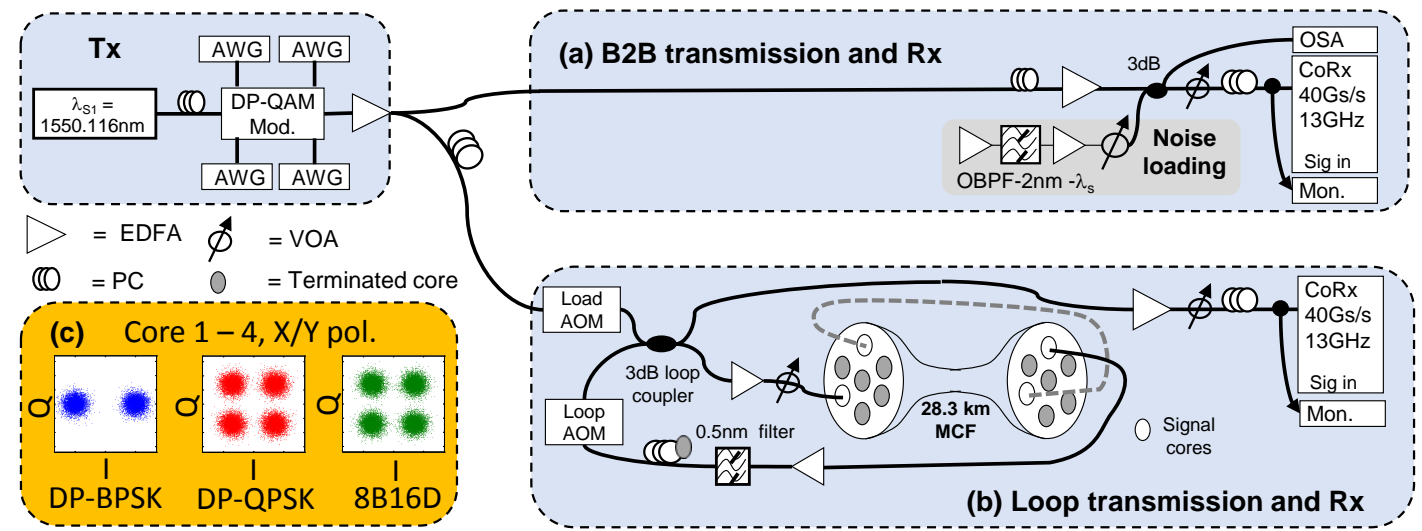

Fig. 1: Experimental transmission setup for the (a) back-to-back and (b) recirculating loop experimental evaluation of the 8B16D modulation format and DP-BPSK / DP-QPSK for comparison. (c) Noisy constellation diagrams for the three different modulation formats.

ing a sensitivity benefit. Compared to DP-QPSK, the improvement in $\gamma$ is also $4.77 d B$, but comes at the cost of a $50 \%$ reduction of $S E_{\text {core }}$. The 8B16D modulation described also has a number of potential implementation advantages over other multidimensional formats, especially over those generated by lattice sphere cutting methods $^{3}$. The constellation (shown in Fig. 1 (c)) has a null field average in each core, which facilitates automatic bias settings of the optical modulators and allows the use of AC-coupled receivers. Furthermore, identical constellations on each polarization of each core also eases the polarization demultiplexing mechanism, and it has a constant modulus to enable the use of well-known equalization algorithms, such as the constant modulus algorithm $(\mathrm{CMA})^{10}$. In fact, the field modulation and receiver processing mechanisms required for 8B16D format are similar to DP-QPSK, which simplifies its application and enables format flexible transceivers without significant hardware changes. Hence, 8B16D could be considered as a back-up format to improve sensitivity in degraded links usually carrying DP-QPSK.

\section{Experimental Setup}

The experimental setup for the investigation of the $8 \mathrm{~B} 16 \mathrm{D}$ modulation format is shown in Fig. 1. A $100 \mathrm{kHz}$ linewidth laser operating at $1550 \mathrm{~nm}$ was used as light source for a DP-QAM modulator, driven by four independent arbitrary waveform generators (AWG) operating at $50 \mathrm{GS} / \mathrm{s}$, to generate 25 GBaud signals. Hence, DP-QPSK had a bit rate of $100 \mathrm{Gbit} / \mathrm{s}$, twice that of DP-BPSK or the $8 \mathrm{~B} 16 \mathrm{D}$ modulation format. A fixed symbol rate was chosen to emulate a realistic format flexible transmission system where components such as reconfigurable optical add-drop multiplexers or filters are optimized for a specific symbol rate and the transmission distance adjusted by the choice of modulation format. The setup for required receiver OSNR characterization is shown in Fig. 1 (a). A noise source based on the filtered output of amplified spontaneous emission of erbium-doped fiber amplifiers (EDFAs) followed by a variable optical attenuator (VOA) was combined with the signal to control the receiver OSNR. As only one transmitter was available for the measurements, each of the four cores' signals was transmitted in turn and combined for processing offline. For the recirculating transmission experiment, Fig. 1 (b), the signals were transmitted in series through two cores of a $28.35 \mathrm{~km}$ long 7-core MCF for each $56.7 \mathrm{~km}$ recirculation with acousto-optic modulators (AOMs) used to control the loading and recirculation times. Both AOMs were driven by a timing controller, which was also used to gate the receiver for measurements as a function of transmission distance. The optimum input power at the beginning of each loop recirculation was measured to be $-2 d B m$. Again, due to equipment limitations, each core-specific data sequence was transmitted sequentially and combined for postprocessing. The signals were received on a polarization-diversity coherent receiver with a 100 $\mathrm{kHz}$ local oscillator laser and sampled at $80 \mathrm{GS} / \mathrm{s}$ with a digital sampling oscilloscope. Digital signal processing was performed offline using MATLAB. It consisted of resampling, orthonormalization and frequency-domain dispersion compensation stages, followed by timing recovery at 4 samples per symbol. The resulting signal was then resampled to 2 samples per symbol and sent to a multiple-input multiple-output (MIMO) equalizer for polarization de-multiplexing. The MIMO equalizers were 17-tap digital filters updated using a decision-directed least mean squares (DD-LMS) algorithm, with carrier recovery within the equal- 
izer loop. The equalizer taps were initially set by updating the taps using CMA and switching to DD-LMS after convergence. The 4-dimensional signals from each core were then combined to form a 16-dimensional signal for decoding and error counting. We note that alternative decoding strategies, with simpler receiver structure, exist ${ }^{11}$, but for simplicity, a maximum likelihood decoder was implemented to detect the 16-dimensional constellation points. BER measurement of the 8B16D modulation format was compared to the average BER of four independent DP-BPSK / DPQPSK signals through the same cores.

\section{Results}

Fig. 2 shows the results of a back-to-back measurement as well as a simulation of the BER vs. the OSNR for DP-QPSK, DP-BPSK, and $8 \mathrm{~B} 16 \mathrm{D}$ at 25 GBaud. DP-QPSK requires the largest OSNR, but it has twice the $S E_{\text {core }}$ of the other formats. DP-BPSK and 8B16D have equal $S E_{\text {core }}$, but DP-BPSK requires an additional 2.5 $\mathrm{dB}$ OSNR over $8 \mathrm{~B} 16 \mathrm{D}$ at a BER of $10^{-3}$.

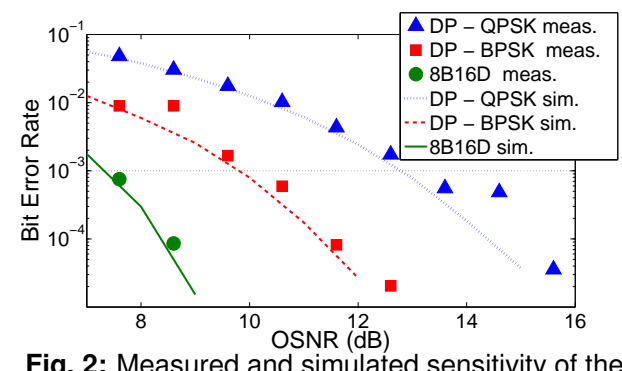

Fig. 2: Measured and simulated sensitivity of the 16-dimensional modulation format that is based on the

Nordstorm-Robinson code, DP-BPSK and DP-QPSK and a reference at $\mathrm{BER}=10^{-3}$. All values are at 25 GBaud.

Fig. 3 shows the BER as a function of the transmission distance for DP-QPSK, DP-BPSK, and 8B16D at 25 GBaud. The DP-QPSK-modulated signal can reach $4500 \mathrm{~km}$ at a $B E R=10^{-3}$, while DP-BPSK can reach up to $9000 \mathrm{~km}$ at the same $\mathrm{BER}$, with the 2 times increase consistent with the expected sensitivity and $S E_{\text {core }}$ difference between them.

For the same $S E_{\text {core }}$ as DP-BPSK, the improved sensitivity of the 8B16D format increases the reach to $14000 \mathrm{~km}$, an increase of $55 \%$, and by over $300 \%$ compared to DP-QPSK. Hence, these results demonstrate that the high sensitivity at high OSNR of the 8B16D format does translate to additional transmission reach in a noise-limited link. Consequently, it is a suitable format for longdistance MCF transmission. Furthermore, it may be useful as a back-up for DP-QPSK in a formatflexible system, when increased transmission distance is required or for degraded links limited by optical noise.

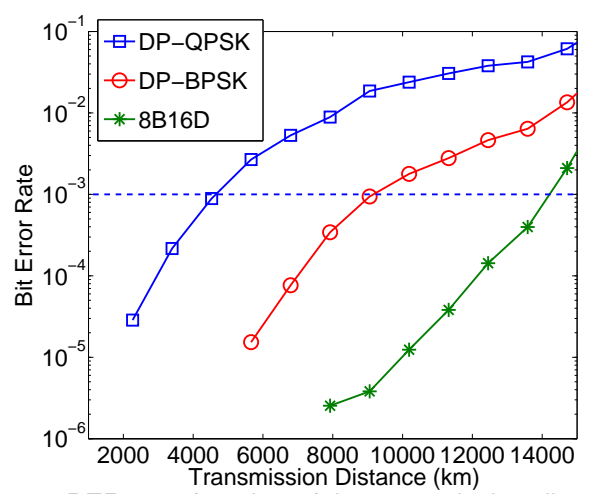

Fig. 3: BER as a function of the transmission distance for DP-QPSK, DP-BPSK and the 8B16D modulation format at 25 GBaud.

\section{Conclusions}

We have experimentally studied a 16-dimensional modulation format with high sensitivity for longhaul fiber optical transmission systems with multicore fibers (MCFs). We observed that for $\mathrm{BER}=10^{-3}$, the modulation format increased the transmission reach by about $55 \%$ at equal spectral efficiency compared to DP-BPSK and by 300 $\%$ compared to DP-QPSK, for half the spectral efficiency. The results show the potential of such formats for long distance and format-flexible transmission. More generally, they show that MCF adoption may improve transmission performance of optical fiber systems beyond a linearly increased capacity with the number of cores.

\section{Acknowledgements}

This work was partially funded by JSPS.

\section{References}

[1] D.J. Richardson et al., "Space-division multiplexing in optical fibres," Nature Photonics, Vol. 7, no. 5, pp. 354-362 (2013).

[2] R. S Luís et al., "Comparing inter-core skew fluctuations in multi-core and single-core fibers," Proc. CLEO, SM3L.5 (2015)

[3] S. O. Arik et al., "High-dimensional modulation for modedivision multiplexing," Opt. Fiber Commun. Conf., p. W4J.1 (2014)

[4] D.S. Millar et al., "High-dimensional modulation for coherent optical communications systems," Optics Express, Vol. 22, no. 7, pp. 8798-8812 (2014).

[5] B.J. Puttnam et al., "Modulation formats for multi-core fiber transmission," Optics Express, Vol. 22, no. 26, pp. 32457-32469 (2014)

[6] E. Agrell et al., "Power-efficient modulation formats in coherent transmission systems," Journal of Lightwave Technology, Vol. 27, no. 22, pp. 5115-5126 (2009).

[7] T.A. Eriksson et al., "Frequency and polarization switched QPSK," Proc. ECOC, p. Th.2.D.4 (2013)

[8] A.W. Nordstrom et al., "An optimum nonlinear code," Information and Control, Vol. 11, no.5, pp. 613-616 (1968).

[9] http://neilsloane.com/packings/index.html

[10] S. Savory, "Digital coherent optical receivers: algorithms and subsystems," J. Sel. Top. Quantum Electron. Vol. 16, no. 5, pp. 1164-1179, (2010).

[11] J.P. Adoul, "Fast ML decoding algorithm for the Nordstrom-Robinson code," IEEE Transactions on Information Theory, Vol. IT-33, no. 6, pp. 931-933 (1987). 